\title{
Experimental demonstration of polarization-assisted transverse and axial optical superresolution
}

\author{
Jason B. Stewart ${ }^{\mathrm{a}, *}$, Bahaa E.A. Saleh ${ }^{\mathrm{a}}$, Malvin C. Teich ${ }^{\mathrm{a}}$, John T. Fourkas ${ }^{\mathrm{b}}$ \\ a Department of Electrical and Computer Engineering, Quantum Imaging Laboratory, Boston University, 8 Saint Marys Street, \\ Boston, MA 02215, USA \\ ${ }^{\mathrm{b}}$ Eugene F. Merkert Chemistry Center, Boston College, Chestnut Hill, MA 02467, USA
}

Received 7 June 2004; received in revised form 16 July 2004; accepted 19 July 2004

\begin{abstract}
A technique has recently been proposed for generating a focused optical beam with focal spot dimensions that are smaller than those of conventional Gaussian beams, in both the axial and transverse directions. The technique is based on superposing two Gaussian beams of orthogonal polarizations with slightly offset foci. As a result of the differential Gouy phase, a $\lambda / 2$ retardation is introduced, thereby creating a polarization component with a superresolved focal volume. This polarization-assisted axial and transverse superresolution effect is experimentally demonstrated here. In agreement with theory, a focal spot-size reduction of more than $30 \%$ is observed in both the axial and transverse directions.
\end{abstract}

(C) 2004 Elsevier B.V. All rights reserved.

\section{Introduction}

Most efforts to enhance optical resolution are based on the use of suitable magnitude and phase pupil filters, which serve to reduce the transverse and axial dimensions of the focal spot [1-6]. We have recently proposed a new polarization-assisted technique that exploits the Gouy phase shift inher-

\footnotetext{
* Corresponding author. Tel.: +1 617353 9961; fax: +1 617 3536440 .

E-mail addresses: jstew@bu.edu (J.B. Stewart), besaleh@ bu.edu (B.E.A. Saleh).

URL: http://www.bu.edu/qil/.
}

ent in focused optical beams [7]. In this paper, we demonstrate experimentally that the polarizationassisted technique operates as promised.

As illustrated in Fig. 1, two orthogonally polarized, coaxial Gaussian beams with axially offset foci $F_{1}$ and $F_{2}$ are superposed. Since the Gouy phase for each beam varies from $-\pi / 2$ to $\pi / 2$, and exhibits a value of zero at the beam focus [8], the differential Gouy phase between the two beams is zero everywhere except near the foci, where it reaches a maximum value of $\pi$ at the center of the beams (the mid point $\mathrm{C}$ between the offset foci). If the beams are of orthogonal linear 


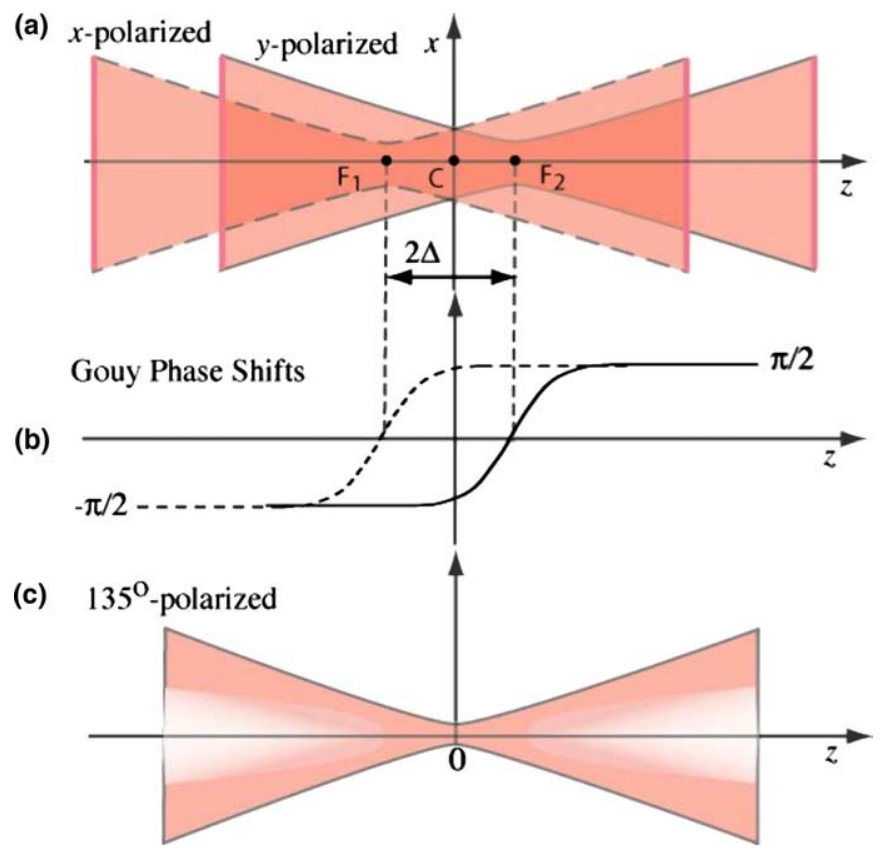

Fig. 1. (a) Two orthogonally polarized Gaussian beams with offset foci $F_{1}$ and $F_{2}$ are superposed. At points far from the foci, the $x$ and $y$-polarized components are in phase at the beam axis. (b) The difference between the Gouy phase shifts associated with the two beams attains a maximum value of $\pi$ at the center C. (c) The $135^{\circ}$ linear-polarization component (minority) is highly confined in the focal region. It has an annular intensity distribution away from the center.

polarization at angles $0^{\circ}$ and $90^{\circ}$, they incur a retardation confined to the region near the foci, with a maximum value corresponding to a halfwave at the center. The polarization state of the combined beams changes dramatically in the region near the foci. If the beams are in phase at axial points far from the foci, they add up to a linearly polarized component at $45^{\circ}$, called the majority polarization, but they are converted to an orthogonal linear polarization at $135^{\circ}$, called the minority polarization.

The minority component forms a non-Gaussian beam with unique axial and transverse characteristics. It vanishes at all axial points except near the center. Away from the center, it has an off-axis transverse annular structure created by the differential off-axis wavefront curvature between the constituent Gaussian beams. As is well known, such an annular distribution corresponds to stronger transverse confinement in the focal region. The minority-polarization beam therefore, has a focal spot smaller than that of the constituent Gaussian beams in both the transverse and axial directions, as demonstrated theoretically in [7]. When used as illumination in a scanning imaging system, such as a scanning microscope, a superresolved image is produced.

\section{Experiment}

A Gaussian beam is used to generate two orthogonally polarized coaxial Gaussian beams with foci offset [from the center] by a distance $\Delta$. The transverse and axial distributions of the intensity of the component with linear polarization at $135^{\circ}$ (the minority beam) are to be measured at points within the focal volume for different offset distances.

As illustrated in Fig. 2, the experimental arrangement used to generate and control the displaced Gaussian beams is based on a Mach-Zehnder interferometer geometry [8] in which polarizing beam splitters are used to separate, and then 


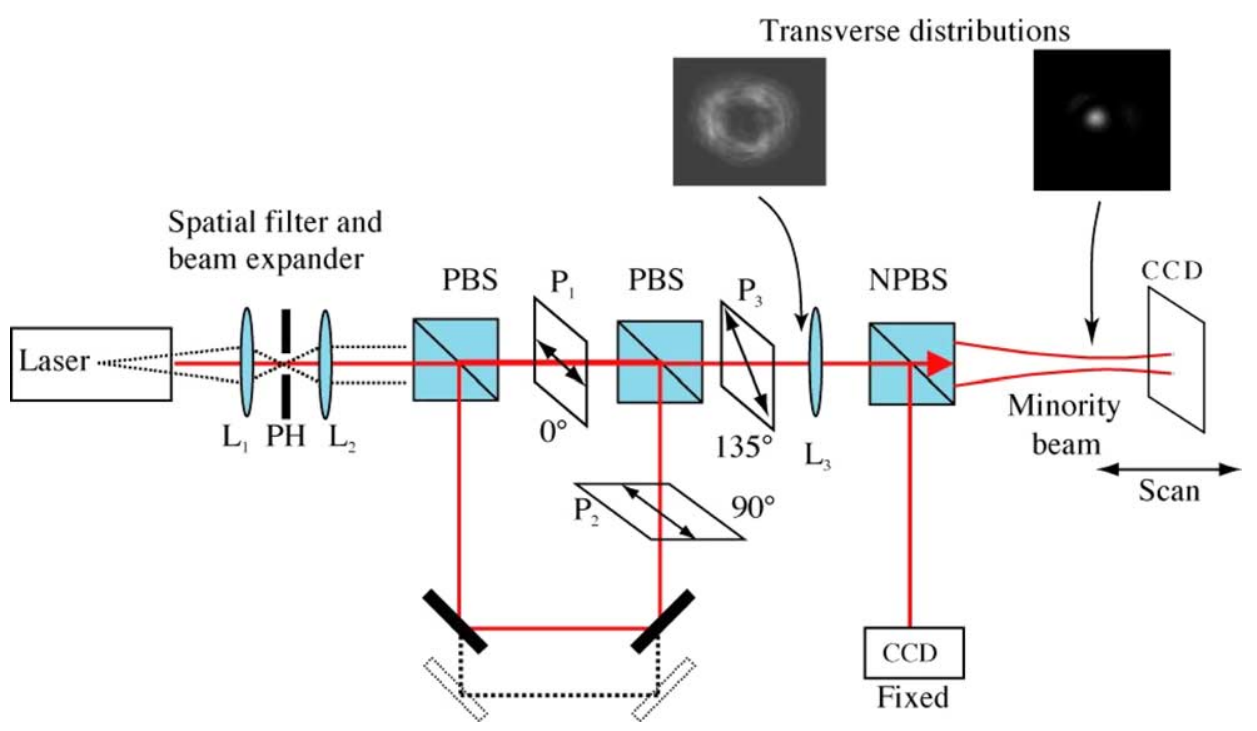

Fig. 2. Experimental arrangement: L, lens; PH, pinhole; PBS, polarizing beam splitter; P, polarizer; NPBS, non-polarizing beam splitter; CCD, charge-coupled-detector array camera. Polarizer angles are measured with respect to the horizontal. At lens $\mathrm{L}_{3}$, the $x$ and $y$-polarized beams have approximately equal widths, but slightly different wavefront curvatures, so that their foci are separated by an offset distance $\Delta$ that is controlled by the interferometer path length difference. Polarizer $\mathrm{P}_{3}$ selects the minority polarization beam.

recombine, the $x$ - and $y$-polarized beams after they travel slightly different distances and thereby acquire different wavefront curvatures. The recombined beams, which are of approximately the same diameter, are focused by a lens to two different foci. The offset distance $\Delta$ is changed by varying the optical path difference in the interferometer, using a nano-positioning stage with 10-nm stepping resolution. A Glan-Thompson linear polarizer $\left(\mathrm{P}_{3}\right)$ at $135^{\circ}$ is used to select the minority polarization, which is imaged by a 1 -m focal length lens $\left(\mathrm{L}_{3}\right)$ onto a $640 \times 480$ pixel scanning CCD array. A long focal-length lens is employed to insure that the transverse spot distribution is sampled by many pixels of the CCD camera. The CCD array is translated in millimeter steps along the axis of the beam, recording its transverse intensity profile at each axial location.

The light source used in the experiment is a randomly-polarized $\mathrm{He}-\mathrm{Ne}$ laser with a HermiteGaussian TEM $_{00}$ transverse mode and a central wavelength of $632.8 \mathrm{~nm}$. The beam passes through a spatial filter and beam-expander assembly, which expands the beam by a factor of five, collimates it, and filters out any spatial nonuniformity. To maintain the Gaussian nature of the beam, the aperture sizes of the lenses used in the assembly are much larger than the diameter of the beam. The focal lengths selected for lenses $L_{1}$ and $L_{2}$ are 16 and $75.6 \mathrm{~mm}$, respectively. The spatial-filter pinhole $\mathrm{PH}$ diameter is $50 \mu \mathrm{m}$.

Two linear polarizers with greater than 10,000:1 extinction ratios $\left(\mathrm{P}_{1}\right.$ and $\left.\mathrm{P}_{2}\right)$ are placed before the recombining polarizing beam splitter to reduce any elliptical polarization phase shifts introduced by the first beam splitter or reflections from the mirrors (for the $y$-polarized beam). The polarizer rotation angle coincides with the polarization direction of the $x$ - and $y$-polarized beams in the two arms of the interferometer.

A non-polarizing beam splitter and a fixed CCD array are used to monitor the phase difference between the $x$ - and $y$-polarized beams. If a phase shift between the interferometer arms is observed, the nano-positioning stage is moved to correct for the phase shift.

The measured transverse and axial distributions are post-processed and analyzed using a Matlab program in which they are smoothed using an averaging filter, normalized and then assembled 
to form a 3-dimensional representation of the minority-beam intensity distribution. The fullwidth half-maximum (FWHM) of the distribution is recorded in both the transverse and axial directions.

\section{Results}

To determine the level of resolution enhancement achievable with the experimental arrangement displayed in Fig. 2, a numerical analysis of the system was carried out. The propagation of two $x$ - and $y$-polarized paraxial Gaussian beams through a similar system with ideal optics was modeled using a Matlab program. The path length of the $y$-polarized arm of the interferometer was varied and its effect on the transverse and axial resolution of the minority-polarized beam was calculated. These results were used to select an optimum optical path length difference between the interferometer arms for demonstrating superresolution.

Superresolution is gauged by comparing the resolution of the minority-polarized beam to that of a conventional Gaussian beam. In the data presented, the $x$-polarized beam is selected to represent the resolution of a conventional beam, although the $y$-polarized beam could equally well have been used. Superresolution in both the transverse and axial directions is quantified by determining the ratio of the minority-beam FWHM to the conventional-beam FWHM, a quantity we define as the resolution-enhancement factor $G$.

The resolution enhancement experienced by the minority beam in the axial direction is shown in Fig. 3. As mentioned above, the data recorded for the $x$ - and minority-polarized beams are processed by a Matlab routine that first filters the intensity data captured by the CCD camera and then finds the peak intensity value for each image along the beam axis. This represents the axial intensity distribution of the beam. Analysis of these profiles is performed with separate plotting software. The axial intensity profile of the $x$-polarized beam was fit to a Lorentzian distribution (dashed curve) with a FWHM of 2.85. The axial intensity data for the minority-polarized beam (triangles) were fit to a theoretical curve with a FWHM of 1.99. This corre-

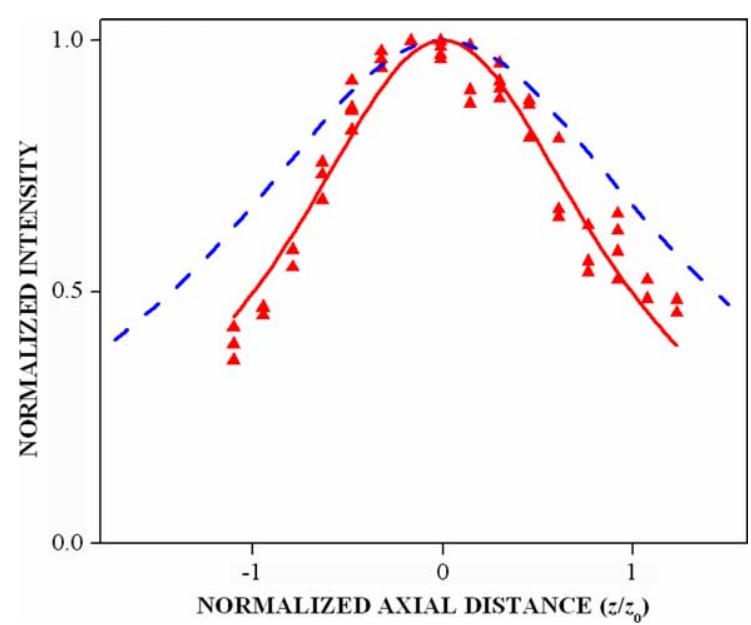

Fig. 3. Axial distribution of the minority polarized beam (triangles represent multiple positions of the CCD plane recorded on multiple passes), theoretical fit (solid curve), and the constituent linearly polarized Gaussian beam (dashed curve), for an offset parameter $\Delta / z_{0}=0.62$. The measured resolution enhancement factor $G=0.6962 \pm 0.0432$, compared to a calculated value of $G_{\text {theory }}=0.6346$.

sponds to $G=0.6962 \pm 0.0432$, a $30 \%$ enhancement in axial resolution. The calculated enhancement factor for this experimental arrangement was $G_{\text {theory }}=0.6346$.

The variability in the axial superresolution data arises principally from fluctuations in the phase of the two polarized beam components between CCD frame captures. To minimize this problem, a photodiode-based feedback system could be used in the interferometer to measure and correct for phase fluctuations by adjusting a nano-positioning stage or a mirror mounted on a piezoelectric device. Unfortunately, communication with the nano-positioning stage used in this experiment was not sufficiently fast to be used for feedback purposes.

The transverse resolution of a beam is greatest at its waist. Therefore, the normalized transverse superresolution data provided in Fig. 4 present a comparison of the minority-polarized beam waist and the $x$-polarized beam waist, both of which occur at different distances from the objective lens. The transverse intensity profile of the $x$-polarized beam was fit to a Gaussian distribution (dashed curve) with a FWHM of 1.78. The transverse intensity data for the minority-polarized beam (tri- 


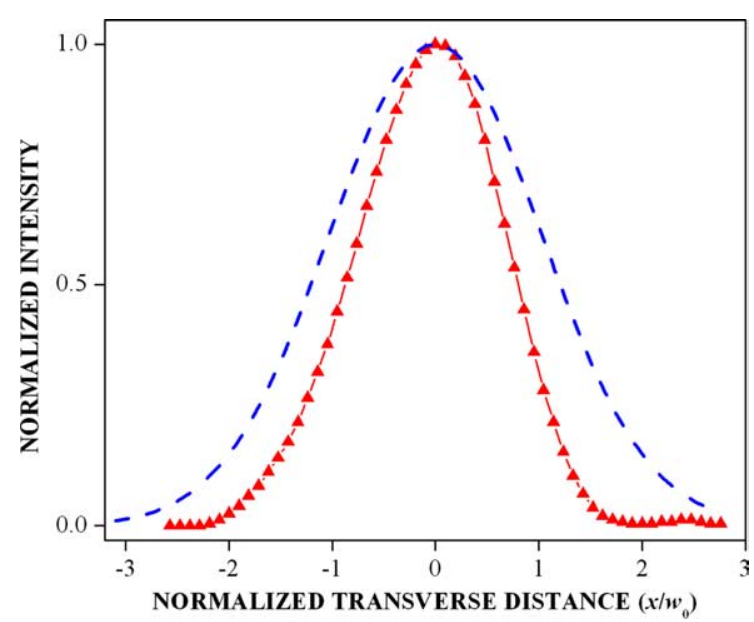

Fig. 4. Transverse distribution of the minority-polarized beam (triangles/solid curve, measured halfway between the constituent beams, at location C, in Fig. 1(a)) and the constituent linearly polarized Gaussian beam (dashed curve, measured at the focal plane, $F_{1}$, in Fig. 1(a)). The data correspond to an offset parameter $\Delta / z_{0}=0.62$. The measured resolution enhancement factor $G=0.6584 \pm 0.0078$, in comparison with a calculated value $G_{\text {theory }}=0.6857$.

angles) was also fit to a theoretical curve, with a FWHM of 1.17. This corresponds to $G=0.6584 \pm 0.0078$, a $34 \%$ enhancement in transverse resolution. The calculated enhancement factor for this experimental arrangement was $G_{\text {theory }}=0.6857$. As a result of non-ideal optics, the transverse profiles of the $x$ - and minority-polarized beams do not exhibit the symmetry about the beam axis that is expected for the ideal case. The intensity profiles displayed in Fig. 4 therefore correspond to the values for a row of CCD pixels through the peak of the transverse intensity distribution. This profile is in the same CCD direction for both beams.

\section{Summary}

Polarization-assisted transverse and axial superresolution have been demonstrated experi- mentally. This technique relies on the superposition of orthogonally polarized Gaussian beams with different wavefront curvatures, which serves to produce a non-Gaussian beam with superresolved waist dimensions. A resolution enhancement of $30 \%$ in the axial direction and $34 \%$ in the transverse direction was attained, relative to a conventional Gaussian beam, in good agreement with theoretical predictions. The enhancement of axial and transverse resolution is also currently being pursued using more elaborate methods. The benefits of using radially polarized beams, for example, have recently been reported [9].

\section{Acknowledgements}

This work was supported by the National Science Foundation (Grant ECS-0088438); the Center for Subsurface Imaging Systems (CenSSIS), an NSF Engineering Research Center; and the David \& Lucile Packard Foundation. JTF is a Research Corporation Cottrell Scholar and a Camille Dreyfus Teacher-Scholar.

\section{References}

[1] T.R.M. Sales, Phys. Rev. Lett. 81 (1998) 3844.

[2] W. Lukosz, J. Opt. Soc. Am. 56 (1966) 1463.

[3] W. Lukosz, J. Opt. Soc. Am. 57 (1967) 932.

[4] M. Martinez-Corral, P. Andrés, J. Ojeda-Castañeda, G. Saavedra, Opt. Commun. 119 (1995) 491.

[5] M. Martinez-Corral, P. Andrés, C.J. Zapata-Rodríguez, M. Kowalcyzk, Opt. Commun. 165 (1999) 267.

[6] T.R.M. Sales, G.M. Morris, Opt. Commun. 156 (1998) 227.

[7] A.I. Whiting, A.F. Abouraddy, B.E.A. Saleh, M.C. Teich, J.T. Fourkas, Opt. Exp. 11 (2003) 1714, Available from: http://www.opticsexpress.org/abstract.cfm?URI=OPEX-1115-1714.

[8] B.E.A. Saleh, M.C. Teich, Fundamentals of Photonics, Wiley, New York, 1991.

[9] R. Dorn, S. Quabis, G. Leuchs, Phys. Rev. Lett. 91 (2003) 233901. 